\title{
XIII.
}

Aus der Hals-, Nasen- und Ohrenklinik an der K. MilitärMedizinischen Akademie zu St. Petersburg (Vorstand: Professor

N. P. Simanowsky).

\section{Ueber einige paradoze Fälle bei der funktionellen Prüfung des Labyrinths ${ }^{1}$ )}

\author{
Von \\ Dr. W. Wojatschek, 2. Assistent.
}

(Mit 7 Curven).

Es wird in letzter Zeit recht oft von Beobachtungen berichtet, die mit den allgemein verbreiteten Ansichten tiber das Wesen der nicht akustischen Labyrinthfunktion gleichsam nicht im Einklange stehen. Die einen von ihnen können fast gar nicht befriedigend gedeutet werden, die anderen stehen so zu sagen nur seheinbar mit den herrschenden Theorien im Widerspruche. $\mathrm{Zu}$ solchen paradoxen Fällen muß ich einige Beobachtungen zählen, welche in der Klinik meines hochrerehrten Chefs Prof. N. P. Simanowsky gemacht und zu seiner Zeit in den Sitzungen der Gesellsebaft der Hals-, Nasen- und Obrenärzte zu St.-Petersburg mitgeteilt wurden.

Die erste Beobachtung (Dr. S. I. Belin of s) betraf eine Kranke, bei der eine eigentimliche Reaktion der Labyrinthe auf Veränderung des Druckes im äußeren Gehörgange festgestellt war. Wir wissen, daß bei eitrigen Otitiden mitunter dureh Luftverdichtung oder Luftverdinnung im äußeren Gehörgange eine Reizung der Bogengänge hervorgerufen werden kann, aber in solchen Fällen äußert sich die Labyrinthreaktion meistenteils durch Sehwindel und Nystagmus. Der letztere hat alle Eigensehaften des rhythmischen Nystagmus und verändert seine

1) Mitgeteilt in der Gesellschaft der Hals-, Nasen- und Ohrenărzte zu St. Petersburg am 10. (23.) Mai 1908. 
Richtung, wenn der Druek negativ wird. In dem Falle Dr. Belinofs (otosklerosis primaria) beobachtete man etwas ganz Eigenartiges. Beim Zusammendrticken der Luft im åußeren Gehörgange machten die Augäpfel der Patientin eine einmalige Wendung in der sagittalen Ebene, die freilich nicht großaber deutlich zu sehen war; dabei bewegten sich die Corneae, soviel ich mich erinnere, von oben nach unten. Kehrte der Druck zur Norm zurlick, so gingen die Augen auch zurlick, d. i. in ihre ursprüngliche Lage. Hier bestand also die Eigentümliehkeit darin, daß die Augäpfel auf Reizung des Labyrinths nicht durch mehrmalige Zuckungen, wie es gewöhnlich beim Nystagmus der Fall ist, sondern dureh eine einmalige Wend ung reagierten.

In einem anderen analogen Falle zeigte ein Kranker Dr. A. J. Galebskys mit einem Trommelfelldefekt und inveterierter Otorrhoe bei Versuchen mit Vergrößerung und Verminderung des Druckes auch einen ebensolchen Reflex an den Augen, aber nur in einem geringeren Grade.

Durch die erwähnten einmaligen Wendungen des Augapfels wird bekanntlich die Funktion jener Labyrinthteile, welche auf sogenannte progressive Beschleunigung reagieren können, gekennzeichnet. Letztere Beschleunigung wird der Winkelbeschleunigung, die als natürlicher Reiz der Ampullen der Bogengänge gilt, entgegengestellt. Uns ist der sogenannte Mechanismus des Empfindens der progressiven Beschleunigung noch nicht genau bekannt, wir können aber mit grober Wahrscheinlichkeit annehmen, daß diese Funktion in den Vorhofssäckehen lokalisiert sei. Als ein Beispiel der progressiven Beschleunigung dient die Gravitationsbeschleunigung. Diese Beschleunigung bedingt eine unter dem Namen "Gegenrollung des Anges" bekannte Erscheinung, die darin sich ausdrückt, daß beim Beugen des Kopfes in der frontalen Ebene der Augapfel nach der dieser frontalen Ebene entgegengesetzten Richtung eine einmalige Wendung um einige Grade macht.

Das Vorhandensein eines solchen Reflexes fuhrt uns zur Annahme, daß in den Fällen ron Dr. Belinof und Galebsky, wo aucb einmalige Wendungen des Auges in einer bestimmten Ebene stattfanden, wir es gerade mit einer Reizung des Sacculus und Utriculus, die auf progressive Beschleunigung reagieren, zu tun batten. Bei der theoretischen Erwägung kann man sich leicht vorstellen, daß bei der Sklerose, also bei abnormer Regulierung 
des intralabyrinthären Druckes, der von abnormem Zustande des runden oder ovalen Fensters abhängt, Schwankungen des Druckes im äußeren Gehörgange eine atypische Lageveränderung der Flissigkeit im Vorhofe, Verschiebung der Otolithen und daher eine Reizung des entsprechenden Nerven nach sich ziehen könnten; dabei wurde auch zufällig der Apparat gereizt, der seine Tätigkeit unter anderem durch sagittale Wendungen des Auges äufert.

Noch mehr paradox erseheint die Beobachtung Dr. M. F. Zitowitschs. Sein Patient mit otitis media purulenta reagierte auf die Kalorisation folgendermaßen: bei Einspritzung kalten Wassers ins rechte Ohr erhielt man einen reinen horizontalen Nystagmus nach links ohne Beimengung von Rotationskomponenten. Bei Anwendung von heißem Wasser kam gleichfalls nur ein horizontaler Nystagmus aber nach der entgegengesetzten Richtung, also nach rechts zustande. Diese Eigenttumlichkeit, d. i. das Fehlen des rotatorisehen $\mathrm{Ny}-$ stagmus konnte leicht dadurch erklärt werden, daß bei diesem Kranken rechts nur der laterale Bogengang funktionierte, während die vertikalen durch ein Cholesteatom oder durch irgend eine andere Ursache außer Tätigkeit gesetzt waren. Es wurde aber bei dem Kranken noch eine andere Eigenart des kalorischen Nystagmus konstatiert: Nach Báránys Regel muß der Nystagmus (nach einer kalten Einspritzung ins rechte Ohr beim Beugen des Kopfes nach links um $90^{\circ}$, seine Richtung verandern, d. $b$. sich in einen horizontalen Nystagmus naeh rechts verwandeln. Diese Veränderung wurde nicht beobachtet: der Nystagmus behielt seine Richtung nach links. - Der Fall Dr. Zitowitsehs ist recht rätselhaft, aber er läßt sich meines Erachtens bis zu einem gewissen Grade auf folgende Weise erklären: die normale Richtungsveränderung des Nystagmus beim Beugen des Kopfes um $90^{\circ}$ kommt daher, daß bei aufrechter Kopfhaltung, d.i. in gewöhnlicher sitzender Lage des Patienten die Ebene des lateralen Bogengangs nach hinten unter einem Winkel von ea. $25^{\circ}-30^{\circ} \mathrm{zum}$ Horizont geneigt ist, und bei Abktiblung der lateralen Labyrinthwand die Lymphe in dem Bogengange sich in der Richtung von der Ampulle zum glatten Ende bewegt (dadurch entstehen horizontale Nystagmuszuckungen nach links) beugt nun der Patient den Kopf um $90^{\circ}$ seitwärts, so liegt der laterale Bogengang beinahe vertikal, und die Lymphströmung. geht in umgekehrter Richtung, d. h. zur Ampulle (dadurch ent- 
steht horizontaler Nystagmus nach rechts) (Dr. Bárány). Im betrachteten Falle konnte die Lymphe nicht frei nach beiden entgegengesetzten Richtungen, sondern nur immer in einer Ricbtung, nämlich von der Ampulle weg, strömen. Es muß hierbei bemerkt werden, daß der horizontale kalorische Nystagmus bei dem Patienten des Dr. Zitowitseh schwach ausgeprägt war, was auf verringerte Beweglichkeit der Lymphe im lateralen Gange überhaupt hinwies. Stellen wir uns vor, das Lumen dieses Ganges wäre durch Cholesteatom so weit verengt, daß er nicht mehr als Kanal funktionieren konnte, die Kupula seiner Ampulle aber bätte ihre Beweglichkeit noch nicht ganz eingebüßt. Dann könnten wir unter solchen Umständen zulassen, daß die Reizung der lateralen Ampulle nicht durch gewöhnliche Kreisbewegungen der Lymphe im Bogengang, sondern nur durch Bewegung der Vorhofslymphe ausgelöst wurde. Diese Bewegungen können in der lateralen Ampulle, welehe unmittelbar in den Vorhof mïndet, auch kleine Versohiebungen der Lymphe hervorrufen, aber die Richtung der Verschiebung könnte diesmal beim Beugen des Kopfes auf 90 o unverändert bleiben.

Recht rätselhaft ist sohließlich auch folgende Beobachtung, die von mir angestellt und dessen Eigentïmlichkeit erst mit Bilfe der photographischen Registrierung erkannt ist.

Bei Untersuchung des Nystagmus bediene ich mich in einigen Fällen der automatischen Registrierung der Augapfelbewegungen, die an der Hand einer einfachen von mir konstruierten Vorrichtung bewerkstelligt wird '). Vermittelst dieses Apparates haben wir die Möglichkeit, alle Zuckungen des Auges beim horizontalen Nystagmus in Form entsprechender Kurven (der rotatorisehe Nystagmus wird mit Hilfe meiner Methode nicht

1) Hier ihre kurze Beschreibung: Auf das untersuchte Auge (bei geschlossenen Augenlidern $/$ wird ein Spiegelchen angebracht, welches das Spiegelbild eines bell erlenchteten (Nernstsche Lampe) vertikalen Spalt auf eine mit Licht empfindlichem Papier überzogene Trommel abspiegelt. Die Trommel ist in ein Gebăude mit horizontalem Spalt eingeschlossen. Bewegt sich der Augapfel oder richtiger gesagt die Cornea, so geht das Spiegelchen hin und her, das abgespiegelte Bild des Spaltes läuft längs dem horizontalen Spalt auf dem Gehăuse, und anf dem Papier wird eine Kurve, welche alle $\mathrm{Be}=$ wegungen des Auges in vergrößertem Maßstabe wiedergibt, abgedruckt.

Eine ausführliche Beschreibung der Methode befindet sich in den "Nachrichten der K. Militär-medizinischen Akademie" (russisch 1908, Mărz) im Artikel "Ueber Nystagmographie" und im "Praktitschesky Wratsch" (russisch - 1908, N. N. 22-24) im Artikel: "Die Bestimmung verschiedener Nystagmusformen mittels graphischer Registrierung derselben." 
registriert) wiederzugeben. Um eine Vorstellung von der Form der Augenbewegungen bei reinem I abyrinthnystagmus zu geben mögen hier Abbildungen zweier Photogramme angefthrt sein: auf der einen (Fig. 1) ist ein dureh Erhöhung des Druckes im

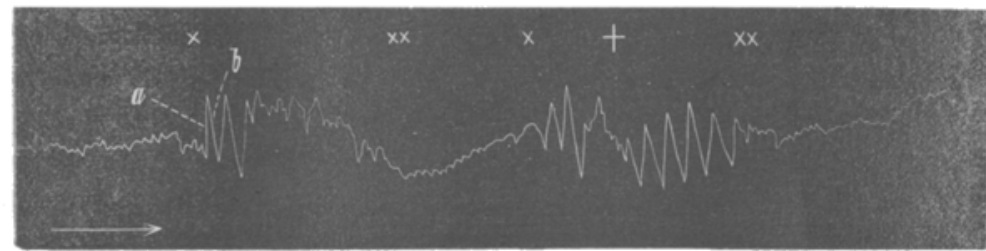

Fig. 1.

äußeren Gehörgang (bei einer Fistel des lateralen Bogengangs) hervorgerufener Nystagmus dargestellt, auf der anderen (Fig. 2) der Nystagmus eines normalen Menschen bei Drehung auf der Zentrifuge. In beiden Fällen zerfallen die einzelnen Nystagmuswellen schroff in zwei Komponenten, eine rasche und eine langsame, was nach Dr. Bárány die typische Eigen-

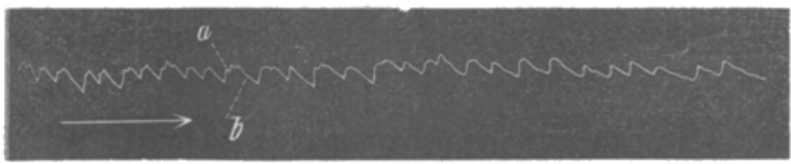

Fig. 2.

tümlichkeit des Labyrinthnystagmus bildet, im Gegensatz zum sogenannten undulierenden Nystagmus, dessen einzelne Wellen aus zwei gleich raseben Komponenten bestehen. Auf dem dritten Photogramm (Fig. 3) ist ein undulierender Nystagmus eines an Gesichtsanomalien leidenden Kranken bei völlig normalen Labyrinthen dargestellt: auf diesem Photogramm sind die

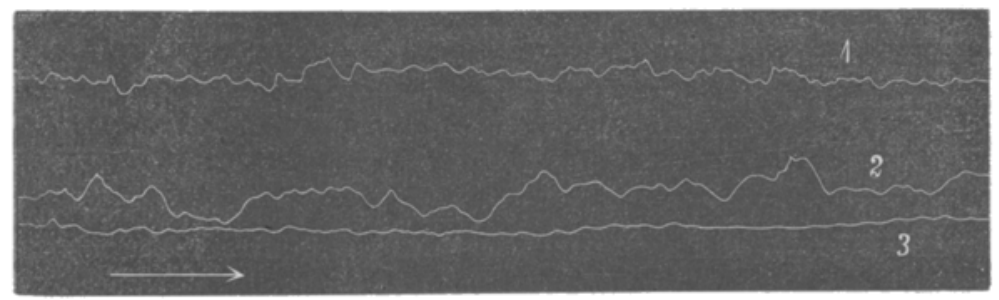

Fig. 3.

Eigenschaften des undulierenden Nystagmus sehr typisch ausgedrückt - der größte Teil der Wellen hat einen gleich steilen Abstieg; zuweilen finden sich freilich Wellen mit ver- 
schiedenen, d. h. nicht gleich-rasehen, Komponenten, aber sie sind regellos verteilt: solch eine Kurve ist ein vollständiges Gegenstück zu der Kurve auf der Fig. 2, auf der die Wellen äußerst regelmäßig sind und alle ausnahmslos einen steilen und einen flachen Schenkel aufweisen 1).

Mein Patient hatte einen spontanen Nystagmus, der duroh seine äußere Merkmale sehr einem spontanen Labyrinthnystagmus glich. Dies ersobien um so wahrscheinlicher, weil der Kranke an einer inveterierten Otitis purulenta litt und wir mit vollem Rechte an ein Labyrinthleiden denken durften. Die Untersuobung mit Hilfe des Photonystagmographen zeigte aber mit Gewißheit, daß in unserem Falle der Nystagmus aus undulierenden Zuckungen bestand und anf diese Weise stellte sich sein nichtlabyrinthärer Ursprung heraus (der Kranke hatte gleichzeitig noch ein Augenleiden). Darin liegt noch gar nichts Außerordentliches, denn man begegnet recht oft Fällen, wo bei Obrenkranken kein Labyrinth-, sondern irgend ein anderer Nystagmus bestimmt wird. Der Nystagmus meines Kranken wies aber noch eine interessante Eigenschaft auf: er veränderte seinen Undulationseharakter bei Reizung des Labyrinths weder durch Drehung noch durch Kalorisation. Dr. Bárány teilt einen Fall mit, in dem bei einem Kranken mit dem undulierenden Nystagmus man anch noch den (rhythmischen) Labyrinthnystagmus hervorrufen, und deswegen eine Kombination beider Nystagmusformen erhalten konnte. In meinem Falle gelang es mir im Gegenteil auf keine Weise den rhythmischen Nystagmus zu erhalten : der Undulationsnystagmus wurde zwar bei Reizung des Labyrinths etwas stärker, behielt aber seinen undulierenden Charakter bei. - Hier folgt die Beschreibung der beiden Versuche, welche zur genauen Untersuchung dieses Falles angestellt worden sind.

1. Der Kranke wird neben dem Nystagmographen gesetzt, dieser Apparat wird an seinem linken Auge, um die Augapfelbewegungen zu registrieren, gestellt. In das rechte $\mathrm{Ohr}$ des Kranken wird eine mit einem Irrigator verbundene Kanüle eingeführt. Eine kalte Kalorisation wird vorgenommen. Allmählioh treten die Symptome der Labyrinthreizung auf:

1) In den zitierten russischen Aufsătzen betrachte ich die Frage der Möglichkeit mittels der Photonystagmographie, in zweifelhaften Fällen den labyrinthären von dem nichtlabyrintbăren Nystagmus zu unterscheiden. 
Schwindel, Nausea, Erbrechen, kalter Sohweib und zuletzt auch Ohnmacht. Trotzdem registriert der in Gang gesetzte Photonystagmograph eine gewöhn liche Kurve mit einem undulierenden Nystagmus

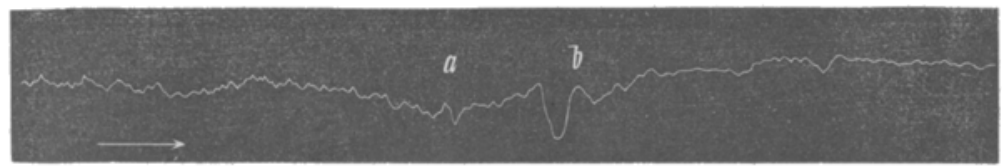

\section{Fig. 4.}

(s. Fig. 4). (Als Gegenstiick bringe ich daneben die Kurve eines anderen Kranken, mit otitis media purulenta, ohne den undulierenden Nystagmus aber, bei dem die kalte Kalnrisation

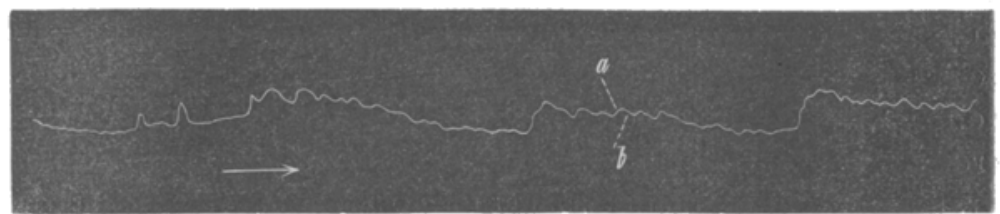

Fig. 5.

schon nach einigen Sekunden $z \mathfrak{u}$ einem äußerst typischen rhythmischen Nystagmus führte [gewöhnlicher Fall] s. Fig. 5).

2. Der Kranke wird auf eine Zentrifuge gesetzt, auf der

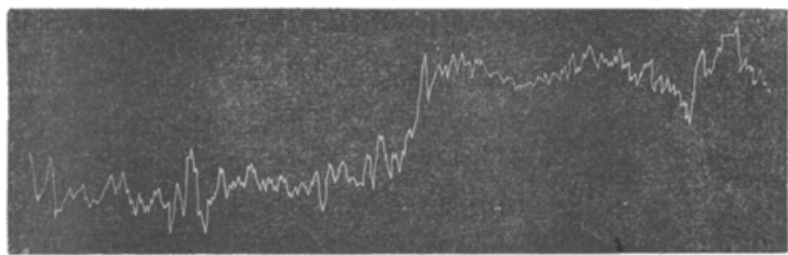

Fig. 6.

auch der Photonystagmograph angebracht wird, der auf das linke Auge eingestellt ist. Es werden 10 Umdrehungen gemacht mit der Geschwindigkeit einer Umdrehung - 4 Sekunden.

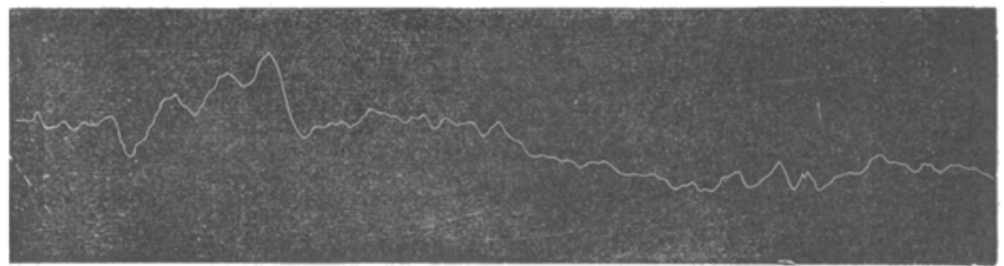

Fig. 7.

Nach dem Anhalten beobachtet man starke Nystagmuszuckungen, welche auch registriert werden. Die erhaltene Kurve (Fig. 6) stellt nur einen undulierenden Nystagmus dar. Der Versuch 
wird wiederholt, dabei wird die Trommel mit dem photographischen Papier, auf dem die Registrierung stattfindet, in doppelt so schnelle Drehung versetzt, damit die einzelnen Details der Wellen (inwieweit die Zeitverhältnisse betrifft) deutlicher ausgedrückt werden. Man erbält ebendasselbe - Fehlen der rhythmischen Zuckungen (s. Fig. 7).

Die beschriebene Erscheinung - die Unmöglichkeit beim Kranken einen Labyrinthnystagmus zu erhalten, kann kaum anders, als auf folgende Weise erklärt werden: bekanntlich zieht die Reizung der Labyrinthe eine ganze Reihe von Symptomen, die in einer bestimmten Reihenfolge nacheinander oder alle gleichzeitig auftreten, nach sich. Stellen wir uns der Einfachheit wegen vor, daß sie nur aus dem Nystagmus, Erbrechen, Sehwindel und den Abwehrbewegungen bestehen und lassen wir zu, daß bei jedem Versuche auf das Labyrinth ein gleich starker Reiz einwirkt. Die Energie des Reizes kann sich auf die einzelnen Symptome ungleichmäßig verteilen; wir wissen z. B., dab bei der horizontalen Drehung und iberhaupt in all den Fällen, wo man beim Untersuchten einen horizontalen Nystagmus hervorruft, die Energie des Reizes in den Nystagmus und das Gefühl der Gegendrehung ibbergeht; Erbrechen aber und andere Symptome kommen selten vor. Bei der Drehung in der vertikalen Ebene, oder überhaupt dann, wenn beim Kranken ein rotatorischer Nystagmus entsteht, erhält man viel leichter Sehwindel, Erbrechen und Abwehrbewegungen, der Nystagmus aber tritt in den Hintergrund. Genaue |Untersuchungen von Dr. Bárảny zeigen, daB in der Regel gerade der rotatorische Drehnystagmus nicht so lang andauert, als der horizontale Drehnystagmus. Hierbei spielt anch die Individualität eine Rolle. Bei ganz gleichen Versuchen beobachten wir bei einem Untersuchten eine äußerst starke Abwehrbewegung und einen schwachen Nystagmus, bei einem anderen keine Neignng zu Abwehrbewegungen aber einen sehr heftigen Nystagmus.

Ich bin der Meinung, daß in der zuletzt angeführten Beobaehtung gerade eine solehe atypische Verteilung der Reizenergie statt hatte: die genannte Energie ging in den Sehwindel, in die Vermehrung der Ausdehnung d. i. Verstärkung einzelner undulierender Nystagmuszuckungen ete. thber, und es bli e b k e in e ubrig, um den rhythmischen Nystagmus zustande zu bringen. 


\section{Erklärung zu den Fig. 1-\%.}

Fig. 1. Nystagmuskurve yon einem an Labyrinthfistel leidenden Kranken. Die Photogramme werden während der Luftdrucksch wankungen im ăußeren Gehörgang aufgenommen.

* Zeitpunkt der Druckerhöhung (große Nystagmuszuckungen: a) rascherer Komponent, b) langsamerer Komponent).

** Rackkehr zum normalen Druck (tritt allmählich auf).

+ Plötzliche Druckschwankung.

Fig. 2. Normale Drehnystagmuskurve.

a) Rascherer Komponent,

b) langsamerer Komponent.

Fig. 3, Ein Beispiel des undulierenden Nystagmus.

1. Beim Blick nach links,

2. und 3. beim Blick nach rechts (zu verschiedenen Versuchsmomenten).

Die Kurve wird aus mehreren unregelmäßigen Wellen zusammengesetzt, welche aber keine Tendenz haben, sich auf zwei Komponenten - einen rascheren und einen langsameren - zu verteilen. Meistenteils sind die betreffenden Wellen aus zwei gleich raschen Komponenten zusammengesetzt.

Fig. 4. Eine andere Kurve von demselben Charakter (undulierendep $\mathrm{Ny}$ stagmus.)

Die Wellen, welche auch eine scheinbare Ähnlichkeit mit den rhythmischen Nystagmuswellen besitzen mögen, folgen nicht in einer regelmäßigen Reihe nacheinander. Bald steht der langsamere Komponent vor dem rascheren (a), bald umgekehrt (b); das kommt bei Labyrinthnystagmus niemals vor. Meistenteils aber ist solche Verteilung nicht ausgeprăgt.

Fig. 5. Kalorische Nystagmuskurve (gewónnlicher Fall). Alle Wellen, so weit es möglich ist, den Unterschied zu bemerken, zerfallen in zwel ungleiche Komponenten, $z$. B.

a) der raschere und

b) der langsamere.

Fig. 6. Eine Kurve des undulierenden Nystagmus. Die Wellen sind verhältnismäßig hoch, behalten aber den undulierenden Charakter (Gleichheit der Komponenten) vollkommen bei.

Fig. 7. Derselbe Fall (die Kurve wird mit verdoppelter Geschwindigkeit der Trommelumdrehungen aufgenommen). 